Abstract PTU-009 Table 1 Median change from Baseline in Key immune, apoptotic and inflammatory markers

\begin{tabular}{|r|c|c|c|}
\hline Median (Q1,Q3) & Baseline & $\begin{array}{c}\Delta \text { From Baseline to 36 } \\
\text { Months OCA (N=161) }\end{array}$ & $\begin{array}{c}\Delta \text { From Baseline to 48 } \\
\text { Months OCA (N=120) }\end{array}$ \\
\hline CRP mg/L & $3(2,7)$ & $-0.7^{* * *}(-2,0.3)$ & $-0.6^{* *}(-2,0.5)$ \\
\hline TNF- $\alpha$ pg/m) & $11(9,14)$ & $-3^{* * *}(-4,-1)$ & $-2^{* * *}(-3,-0.4)$ \\
\hline TGF- $\beta$ ng/mL & $25(19,35)$ & $2(-7,11)$ & $0(-5,7)$ \\
\hline Ck-18 U/L & $124(83,201)$ & $-28^{* * *}(-78,15)$ & $-3(-52,40)$ \\
\hline IL-12 pg/mL & $138(94,198)$ & $9(-21,28)$ & $21^{* *}(-6,43)$ \\
\hline IgA g/L & $2(2,3)$ & $-0.2^{* * *}(-0.5,-0.1)$ & $-0.3^{* * *}(-1,-0.1)$ \\
\hline IgG g/L & $13(11,15)$ & $-1^{* * *}(-2,0.03)$ & $-1^{* * *}(-2,0.2)$ \\
\hline IgM g/L & $4(2,6)$ & $-1^{* * *}(-2,-0.4)$ & $-1^{* * *}(-2,-0.4)$ \\
\hline
\end{tabular}

${ }^{*} \mathrm{p}<0.05 ;{ }^{* *} \mathrm{p}<0.01 ;{ }^{* * *} \mathrm{p}<0.0001$. $\mathrm{p}$-values for the comparisons to baseline were obtained using a Wilcoxon signed rank test. Normal ranges: CRP $0-3 \mathrm{mg} / \mathrm{L}$, TNF- $\alpha$ 8.1 pg/ml, TGF- $\beta$ 18.3-63.4 $\mathrm{ng} / \mathrm{ml}, \mathrm{Ck}-1880 \mathrm{U} / \mathrm{L}$, IL-12 34-246 pg/mL, IgA 0.7-4 g/L, lgG 7-16 g/L, IgM 0.4-2.3 g/L

disease characterized by the immune mediated destruction of bile ducts resulting in cholestasis, cirrhosis and liver failure. The POISE study was a randomized, placebo-controlled phase 3 study investigating daily OCA $5-10 \mathrm{mg}$ in PBC patients with an ongoing open label extension (OLE). The purpose of this analysis was to assess the long-term effects of OCA on key markers of liver damage and inflammation.

Methods Patients enrolled in the double-blind phase had an inadequate response to UDCA or were intolerant of UDCA. Patients were randomized to placebo, OCA 5-10 mg, or OCA $10 \mathrm{mg}$. Upon completion of the 12-month double-blind phase, 198 patients enrolled in the OLE and received OCA. For this analysis, patients were pooled and assessed from the time of first dose of OCA. Data are shown through 48 months of OCA exposure, for patients randomized to placebo in the double-blind phase, only 36 months of data are included.

Results At baseline ( $\mathrm{N}=198)$, patients were 92\% female, mean age was 55 years, PBC disease duration was 9 years, and 93\% received UDCA (mean dose: $16 \mathrm{mg} / \mathrm{kg} /$ day). As observed in the table, there were sustained and significant reductions in immunoglobulins (Ig) A, G and M throughout 48 months of OCA treatment. In addition, significant reductions were observed in tumor necrosis factor (TNF)- $\alpha$, c-reactive protein (CRP), and cleaved cytokeratin (CK)-18 throughout the duration of the OLE. No clinically meaningful changes were observed with transforming growth factor (TGF)- $\beta$ and interleukin (IL)-12, both remained within the normal range.

Conclusions These results demonstrate that OCA has a durable anti-inflammatory effect in patients with $\mathrm{PBC}$ as observed by reductions on key markers of apoptosis (TNF- $\alpha$ and CK-18) and inflammation (CRP). Furthermore, a sustained reduction is observed in IgM, which is classically elevated in PBC patients. The clinical significance of OCA mediated reductions in IgA and $\operatorname{IgG}$ continues to remain unknown.

\section{PTU-010 ROLE FOR GPR15 RATHER THAN BETA 7 INTEGRINS IN THE PATHOGENESIS OF AUTOIMMUNE LIVER DISEASE}

${ }^{1}$ Jonathon Graham*, ${ }^{1}$ Suj Mukherjee, ${ }^{1}$ Muhammed Yuksel, ${ }^{1}$ Rodrigo Liberal, ${ }^{1}$ Giorgina Mieli-Vergani, 'Diego Vergani, 'Y ${ }^{1}$ un Ma, ${ }^{2}$ Bu Hayee. 'Institute of Liver Studies, King's College London, London, UK; ${ }^{2}$ Department of Gastroenterology, King's College Hospital, London, UK
Introduction G protein-coupled receptor 15 (GPR15) is a chemoattractant receptor that directs homing of lymphocytes to the colon. Furthermore, it has been shown to be a mediator of effector $\mathrm{T}$ cell homing during intestinal inflammation. Evidence exists showing infiltration of gut derived $\alpha 4 \beta 7+$ and CCR9+ $\mathrm{T}$ cells in the hepatic infiltrate of patients with autoimmune liver disease (AILD), in particular primary sclerosing cholangitis (PSC), with expression of their complementary ligands also being identified on the hepatic endothelium. However, the role of GPR15 in hepatic gut $\mathrm{T}$ cell homing remains to be defined.

Methods Explanted liver tissue was collected from patients undergoing orthotopic liver transplantation for chronic liver disease (Alcoholic liver disease [ALD] $n=3$, Non-alcoholic fatty liver disease [NAFLD] $n=3$, PSC $n=4$ ) with healthy control tissue sourced from patients undergoing hepatocellular carcinoma (HCC) resection $(n=4)$. Liver infiltrating lymphocytes (LIL) were isolated using a mechanical homogenisation and centrifugation/filtration technique.

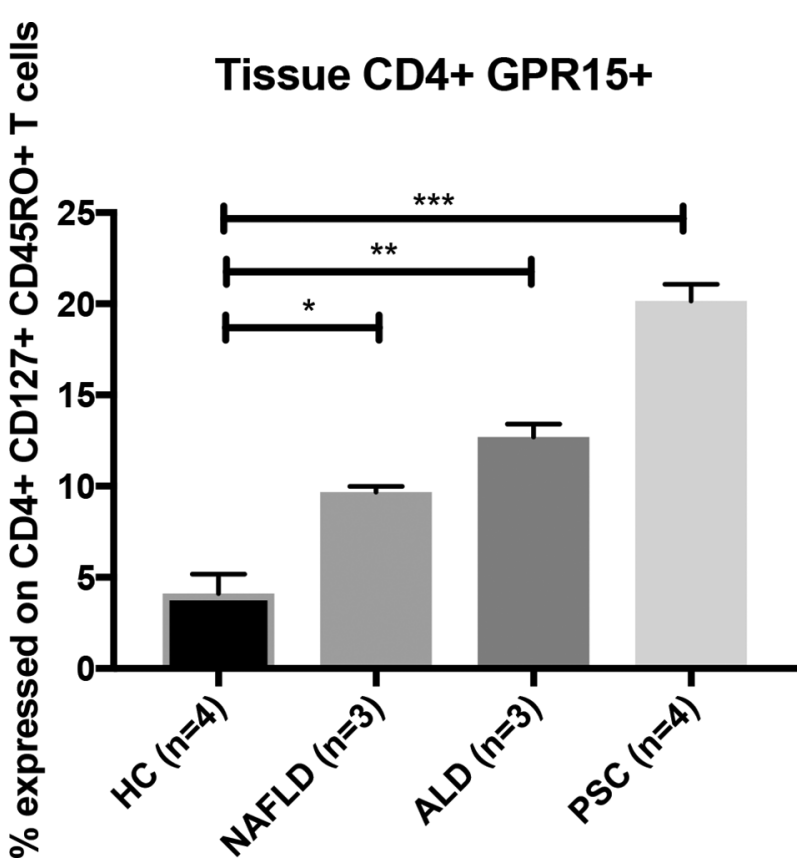

Abstract PTU-010 Figure 1 